\title{
Homoclinic Bifurcations in Planar Piecewise-Linear Systems
}

\author{
Bin Xu, ${ }^{1}$ Fenghong Yang, ${ }^{2}$ Yun Tang, ${ }^{1}$ and Mu Lin ${ }^{2}$ \\ ${ }^{1}$ Department of Mathematical Sciences, Tsinghua University, Beijing 100084, China \\ ${ }^{2}$ Central University of Finance and Economics, School of Applied Mathematics, Beijing 100084, China
}

Correspondence should be addressed to Yun Tang; tangyun@tsinghua.edu.cn

Received 25 January 2013; Accepted 29 August 2013

Academic Editor: Rob Sturman

Copyright (C) 2013 Bin Xu et al. This is an open access article distributed under the Creative Commons Attribution License, which permits unrestricted use, distribution, and reproduction in any medium, provided the original work is properly cited.

\begin{abstract}
The problem of homoclinic bifurcations in planar continuous piecewise-linear systems with two zones is studied. This is accomplished by investigating the existence of homoclinic orbits in the systems. The systems with homoclinic orbits can be divided into two cases: the visible saddle-focus (or saddle-center) case and the case of twofold nodes with opposite stability. Necessary and sufficient conditions for the existence of homoclinic orbits are provided for further study of homoclinic bifurcations. Two kinds of homoclinic bifurcations are discussed: one is generically related to nondegenerate homoclinic orbits; the other is the discontinuity induced homoclinic bifurcations related to the boundary. The results show that at least two parameters are needed to unfold all possible homoclinc bifurcations in the systems.
\end{abstract}

\section{Introduction}

Nonsmooth dynamical systems are naturally used to model many physical processes, such as impacting, friction, switching, and sliding systems. The study of the nonsmooth dynamical systems has attracted more and more attention in the recent decades. Piecewise-smooth systems, as an important branch of the nonsmooth dynamical systems, involve collision systems, Filippov systems, higher-order discontinuity systems, and so forth $[1,2]$. In particular, the study of piecewise-linear systems is significantly important because it not only describes some processes such as circuits [3, 4], but also enables us to locally understand the bifurcation phenomena in the nonlinear systems [5].

This paper studies the planar piecewise-linear continuous vector fields with two zones. Without loss of generality, the considered plane is divided into two half-planes by the boundary coinciding with the vertical axis. The system is linear in each of the half-planes and continuous along the vertical axis. In 1998, Freire et al. [6] studied discontinuous bifurcations, proved that there exists at most one limit cycle, and proved that if the limit cycle exists then it is either attracting or repelling, which solved the problem proposed in 1991 by Lum and Chua [3]. di Bernardo et al. [1] studied discontinuity induced bifurcations, including boundary equilibrium bifurcations and grazing bifurcations of limit cycles. Concerning the limit cycle problem, Simpson and Meiss [7] investigated the Hopf branch in this kind of systems and mentioned homoclinic loops in [5]. One can refer to $[8,9]$ for the periodic orbit problems in piecewise-linear systems with multiple nonsmooth boundaries. Bifurcation of limit cycles by perturbing a piecewise-linear Hamiltonian system is considered in $[10,11]$. For the study of bifurcations in piecewise-linear systems with some application problems, see also [12].

In this paper, we further study the existence problem of homoclinic orbits and homoclinic bifurcations in the abovementioned planar piecewise-linear systems. Starting with transforming the system into a canonical form, we found that its homoclinic orbits exist only in two cases: one is the saddle-focus (or saddle-center) system, which is called nondegenerate homoclinic orbits; the other one has two nodes coinciding on the vertical axis which have opposite stability, which is called degenerate homoclinic system. For both cases, the necessary and sufficient conditions for the existence of homoclinic orbits are established and used for the study of the homoclinic bifurcation problem. For the nondegenerate case, we find that there are two kinds of homoclinic bifurcations: 
one is generic, that is, the limit equilibria of the homoclinic orbits digress from the vertical axis; the other is nongeneric, that is, the discontinuity induced homoclinic bifurcation. However, for the degenerate case, only discontinuity induced homoclinic bifurcation occurs. Finally, we point out that at least two parameters are needed to unfold all possible homoclinic bifurcations in the mentioned systems.

The rest of the paper is outlined as follows. In Section 2, we give a brief overview of the concerned system and transform the system into a canonical form. In Section 3, the existence conditions of homoclinic orbits for both nondegenerate and degenerate cases are provided. Then we study the nondegenerate homoclinic bifurcations and the discontinuity induced homoclinic bifurcations in Section 4. Conclusions are drawn in Section 5.

\section{Canonical Forms}

We start with a planar piecewise-linear system:

$$
\dot{\mathbf{x}}= \begin{cases}\widetilde{\mathbf{A}}_{L} \mathbf{x}+\mathbf{b}_{L}, & x_{1} \leq 0 \\ \widetilde{\mathbf{A}}_{R} \mathbf{x}+\mathbf{b}_{R}, & x_{1}>0\end{cases}
$$

where $\mathbf{x}=\left(x_{1}, x_{2}\right)^{T}, \mathbf{b}_{L}, \mathbf{b}_{R} \in \mathbb{R}^{2}$, and $\widetilde{\mathbf{A}}_{L}, \widetilde{\mathbf{A}}_{R}$ are $2 \times 2$ matrices. By the requirement of continuity on the $x_{2}$-axis, we may assume that the system (1) is of the form

$$
\dot{\mathbf{x}}= \begin{cases}\widetilde{\mathbf{A}}_{L} \mathbf{x}+\mathbf{b}, & x_{1} \leq 0 \\ \widetilde{\mathbf{A}}_{R} \mathbf{x}+\mathbf{b}, & x_{1}>0\end{cases}
$$

where

$$
\widetilde{\mathbf{A}}_{L}=\left[\begin{array}{ll}
a_{L} & b \\
c_{L} & d
\end{array}\right], \quad \widetilde{\mathbf{A}}_{R}=\left[\begin{array}{cc}
a_{R} & b \\
c_{R} & d
\end{array}\right], \quad \mathbf{b}=\left[\begin{array}{l}
e \\
f
\end{array}\right] .
$$

Here we call $\widetilde{\mathbf{A}}_{L} \mathbf{x}+\mathbf{b}\left(\widetilde{\mathbf{A}}_{R} \mathbf{x}+\mathbf{b}\right)$ the left (right) half-system of (2) and the region of the plane

$$
D_{L}: x_{1}<0 \quad\left(D_{R}: x_{1}>0\right)
$$

to be the left (right) zone. Suppose that $\operatorname{det} \widetilde{\mathbf{A}}_{i} \neq 0, i=L, R$. An equilibrium $\mathbf{x}^{*}=\left(x_{1}^{*}, x_{2}^{*}\right)^{T}$ of (2), which is of course isolated, is called visible if it is an equilibrium of the left (right) half-system and $x_{1}^{*} \leq 0\left(x_{1}^{*} \geq 0\right)$; otherwise, it is invisible. Let

$$
\mu=b f-d e .
$$

Then it is easy to check that $\mu=0$ if and only if the equilibria of (2) both coincide on the $x_{2}$-axis. We mention that if the equilibrium of one half-system in (2) lies on the $x_{2}$-axis, then by continuity it is also the equilibrium of the other halfsystem.

A homoclinic orbit has both $\alpha$-limit and $\omega$-limit set consisting of one equilibrium, called its limit equilibrium, that with the orbit constitutes the homoclinic loop. A homoclinic orbit is called degenerate if its limit equilibrium lies on the $x_{2}$ axis; otherwise, it is nondegenerate. The following examples for homoclinic orbits describe the two cases.
Example 1. Nondegenerate homoclinic orbits. Take $b=1$, $d=0, e=0$, and $f=1$ in (3).

(a) Set $a_{L}=-0.6333, c_{L}=0.3667, a_{R}=0.5$, and $c_{R}=$ -2 . Then (2) is a saddle-focus system with the nondegenerate homoclinic orbit as shown in Figure 1(a).

(b) Set $a_{L}=0, c_{L}=1, a_{R}=0$, and $c_{R}=-1$. Then (2) is a saddle-center system with the nondegenerate homoclinic orbit as shown in Figure 1(b).

Example 2. Degenerate homoclinic orbit. In (3), we set

$$
\begin{array}{r}
b=1, \quad d=e=f=0, \quad a_{L}=4, \\
c_{L}=-3, \quad a_{R}=-6, \quad c_{R}=-3 .
\end{array}
$$

Then (2) is a node-node system with degenerate homoclinic orbits as shown in Figure 2.

From the above examples, we found that if a homoclinic orbit exists then some of the orbits will cross the $x_{2}$-axis from left to right and some from right to left. That means that the sign of $\dot{x}_{1}=b x_{2}+e$ for the vector field (2) restricted on the $x_{2}$-axis will vary with $x_{2}$. So we should assume that $b \neq 0$. We mention in passing that the existence of a limit cycle for (2) also implies $b \neq 0$ for the same reason. See also [6].

In order to study homoclinic orbits, it is convenient to simplify system (2) to a canonical form.

Lemma 3. For system (2), let $b \neq 0$. Then there exists a linear transformation such that (2) becomes the following canonical system:

$$
\dot{\mathbf{z}}= \begin{cases}\mathbf{f}^{(L)}(\mathbf{z})=\mathbf{A}_{L} \mathbf{z}+\mu \mathbf{e}_{2}, & x \leq 0 \\ \mathbf{f}^{(R)}(\mathbf{z})=\mathbf{A}_{R} \mathbf{z}+\mu \mathbf{e}_{2}, & x>0\end{cases}
$$

where

$$
\begin{array}{r}
\mathbf{z}=\left[\begin{array}{l}
x \\
y
\end{array}\right], \quad \mathbf{e}_{2}=\left[\begin{array}{l}
0 \\
1
\end{array}\right], \quad \mathbf{A}_{i}=\left[\begin{array}{cc}
\tau_{i} & 1 \\
-\delta_{i} & 0
\end{array}\right], \\
i=L, R .
\end{array}
$$

$\tau_{i}$ and $\delta_{i}(\neq 0)$ are, respectively, the trace and determinant of the matrixes $\mathbf{A}_{i}, i=L, R$, and $\mu$ is given by (5), that is, $\mu=b f-d e$.

Proof. For (2),

$$
\begin{array}{r}
\dot{x}_{1}=a_{i} x_{1}+b x_{2}+e, \\
\dot{x}_{2}=c_{i} x_{1}+d x_{2}+f, \\
i=L, R .
\end{array}
$$

Set the linear transformation

$$
x=x_{1}, \quad y=-d x_{1}+b x_{2}+e .
$$

Equation (9) then becomes

$$
\begin{gathered}
\dot{x}=\left(a_{i}+d\right) x+y=\tau_{i} x+y, \\
\dot{y}=\left(-a_{i} d+c_{i} b\right) x+(b f-d e)=-\delta_{i} x+\mu, \\
i=L, R .
\end{gathered}
$$




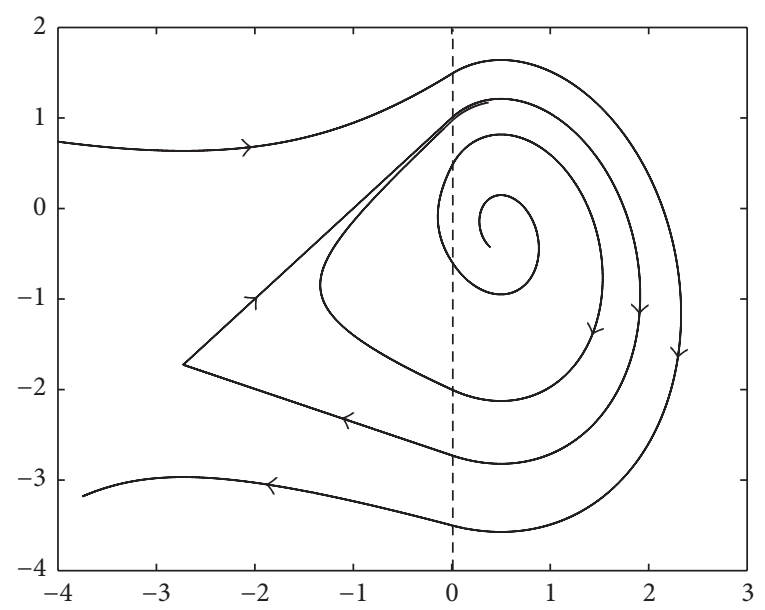

(a)

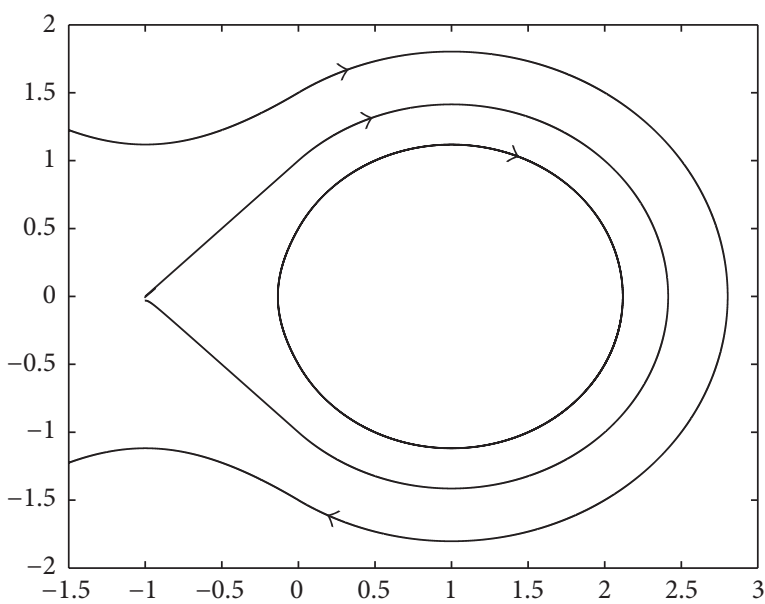

(b)

FIGURE 1: (a) Nondegenerate homoclinic orbit for the saddle-focus system. (b) Nondegenerate homoclinic orbit for the saddle-center system.

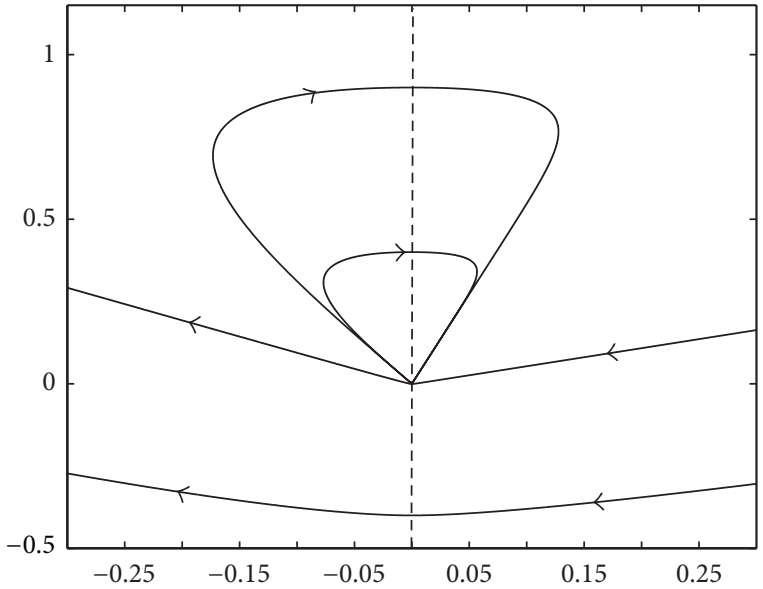

FIGURE 2: Degenerate homoclinic orbits for the node-node system.

Remark 4. The canonical form (7) was mentioned in Simpson [5] by introducing the observability concept. Here we only need the condition $b \neq 0$ instead.

Remark 5. The equilibria of system (7) are

$$
\mathbf{z}_{i}^{*}=\left[\begin{array}{c}
x_{i}^{*} \\
y_{i}^{*}
\end{array}\right]=\frac{\mu}{\delta_{i}}\left[\begin{array}{c}
1 \\
-\tau_{i}
\end{array}\right], \quad i=L, R
$$

From (7), it follows that $\mathbf{z}_{L}^{*}\left(\mathbf{z}_{R}^{*}\right)$ is visible if and only if

$$
\mu \delta_{L} \leq 0 \quad\left(\mu \delta_{R} \geq 0\right) .
$$

In particular, $\mu=0$ if and only if both equilibria of (7) coincide at the origin.

Remark 6. Write

$$
\begin{gathered}
\Sigma=\left\{(0, y)^{T}: y \in \mathbb{R}\right\}, \quad \Sigma^{+}=\left\{(0, y)^{T}: y>0\right\}, \\
\Sigma^{-}=\left\{(0, y)^{T}: y<0\right\} .
\end{gathered}
$$

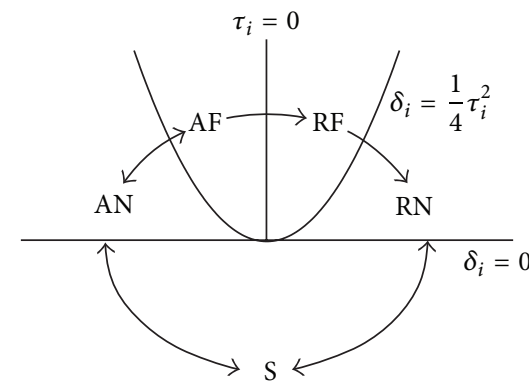

Figure 3: The trace-determinant plane.

We see that, on the $y$-axis, $\Sigma$, the first component of the vector field (7), becomes $\dot{x}=y$. So any orbit of (7) crossing $\Sigma^{+}$ is from left to right, and crossing $\Sigma^{-}$is from right to left. Moreover, for $\mu \neq 0$, the orbit crossing the origin is tangent to the $y$-axis $\Sigma$ at the origin.

Remark 7. The eigenvalues at the equilibria (12) are

$$
\lambda_{1,2}=\frac{\tau_{i}}{2} \pm \sqrt{\frac{1}{4} \tau_{i}^{2}-\delta_{i}}, \quad i=L, R .
$$

We may then classify each half-system of (7) by $\tau_{i}$ and $\delta_{i}$ in the trace-determinant plane as shown in Figure 3. There are 5 types of linear systems: saddle (S), attracting node (AN), repelling node $(\mathrm{RN})$, attracting focus $(\mathrm{AF})$, and repelling focus (RF).

Remark 8. Lemma 3 can be promoted to high-dimensional case; see [5].

To end this section, we introduce two propositions [6].

Proposition 9. System (7) has at most one limit cycle or homoclinic orbit. It is either attracting or repelling. 
Proposition 10. If system (7) has a limit cycle or a homoclinic loop, then $\tau_{L} \tau_{R} \leq 0$.

\section{Existence of Homoclinic Orbits}

We focus on conditions for system (7) to have homoclinic orbits in this section. The homoclinic orbits can be divided into two classes: nondegenerate homoclinic orbits for visible saddle-focus (or saddle-center) systems and degenerate homoclinic orbits for twofold node systems.

\subsection{Nondegenerate Case}

Theorem 11. Let (7) have a nondegenerate homoclinic orbit $\Gamma$. Then (7) is a saddle-focus (or saddle-center) system with a visible saddle in one zone and a visible focus (or center) in the other zone.

Proof. Without loss of generality, we assume that the limit equilibium of $\Gamma$ is in the left zone, denoted by $\mathbf{z}^{*(L)}$. Due to piecewise-linearity of (7), the orbit $\Gamma$ starting from $\mathbf{z}^{*(L)}$ should cross the positive $y$-axis $\Sigma^{+}$to enter the right zone and then back to the original $\mathbf{z}^{*(L)}$. So $\mathbf{z}^{*(L)}$ is a visible saddle in the left zone. By the generalized Poincaré-Bendixon theorem [13], inside the homoclinic loop of $\Gamma$ there is one equilibrium $\mathbf{z}^{*(R)}$, which is a visible focus (or center) in the right zone. So (7) is a saddle-focus (or saddle-center) system.

Remark 12. From Remark 5, it easy to see that the existence of nondegenerate homoclinic orbits implies $\mu \neq 0$. Moreover, the saddle of (7) as the limit equilibrium of $\Gamma$ is in the left zone for $\mu>0$ and in the right zone for $\mu<0$.

The following theorem gives conditions for the existence of nondegenerate homoclinic orbits.

Theorem 13. Let system (7) be a saddle-focus (or saddlecenter) system with a visible saddle in one zone and a visible focus (or center) in the other zone. Denote by $\lambda_{1}>0>\lambda_{2}$ the eigenvalues of the saddle half-system and by $\alpha \pm i \beta, \beta>0$, the eigenvalues of the focus (or center) half-system. Write

$$
\begin{array}{r}
\Lambda=\frac{1}{2} \ln \frac{\lambda_{2}^{2}\left(\lambda_{1}^{2}-2 \alpha \lambda_{1}+\alpha^{2}+\beta^{2}\right)}{\lambda_{1}^{2}\left(\lambda_{2}^{2}-2 \alpha \lambda_{2}+\alpha^{2}+\beta^{2}\right)} \\
-\frac{\alpha}{\beta}\left(2 \pi-\arctan \frac{\lambda_{2} \alpha-\left(\alpha^{2}+\beta^{2}\right)}{\lambda_{2} \beta}\right. \\
\left.\quad-\arctan \frac{\alpha^{2}+\beta^{2}-\lambda_{1} \alpha}{\lambda_{1} \beta}\right) .
\end{array}
$$

Then there exists a nondegenerate homoclinic orbit if and only if

$$
\Lambda=0
$$

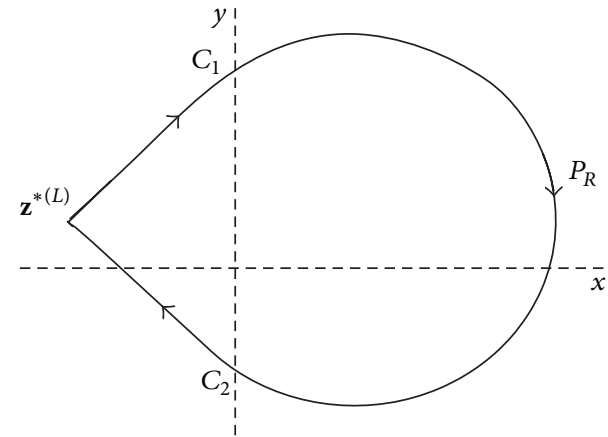

FIgURE 4: A nondegenerate homoclinic orbit.

Proof. We may assume that the saddle of (7) is in the left zone and the focus (or center) is in the right zone, denoted by $\mathbf{z}^{*(L)}$ and $\mathbf{z}^{*(R)}$, respectively. So one has

$$
\delta_{L}<0, \quad \delta_{R}>\frac{1}{4} \tau_{R}^{2}, \quad \mu>0 .
$$

By using (15), the eigenvalues and eigenvectors of the saddle half-system $\mathbf{A}_{L}$ are, respectively,

$$
\begin{gathered}
\lambda_{1,2}=\frac{\tau_{L}}{2} \pm \sqrt{\frac{1}{4} \tau_{L}^{2}-\delta_{L},} \\
v_{1,2}=\left[\begin{array}{c}
1 \\
\left.-\frac{\tau_{L}}{2} \pm \sqrt{\frac{1}{4} \tau_{L}^{2}-\delta_{L}}\right]=\left[\begin{array}{c}
1 \\
-\lambda_{2,1}
\end{array}\right],
\end{array}\right.
\end{gathered}
$$

and the eigenvalues of the focus (or center) half-system $\mathbf{A}_{R}$ are $\alpha \pm i \beta$, where

$$
\alpha=\frac{\tau_{R}}{2}, \quad \beta=\sqrt{\delta_{R}-\frac{1}{4} \tau_{R}^{2}} .
$$

If the nondegenerate homoclinic orbit $\Gamma$ exists, $\Gamma$ will start with the unstable manifold of the saddle $\mathbf{z}^{*(L)}$ to intersect $\Sigma^{+}$ at a point, say, $C_{1}=\left(0, c_{1}\right)^{T}, c_{1}>0$, then along an orbit of the right half focus (or center) system in the right zone to intersect $\Sigma^{-}$at another point, say, $C_{2}=\left(0, c_{2}\right)^{T}, c_{2}<0$, and will finally return along the stable manifold of $\mathbf{z}^{*(L)}$ in the left zone, as shown in Figure 4.

In order to find conditions for the existence of such homoclinic orbit $\Gamma$, we consider the right zone governed by the focus (or center) half-system $\mathbf{f}^{R}(\mathbf{z})$ in (7). Firstly, we transform the right half-system by applying the coordinate transformation

$$
\left[\begin{array}{l}
\tilde{x} \\
\tilde{y}
\end{array}\right]=T^{-1}\left[\begin{array}{l}
x \\
y
\end{array}\right],
$$

where

$$
T=\frac{1}{\beta}\left[\begin{array}{cc}
1 & 0 \\
-\alpha & \beta
\end{array}\right] \text {. }
$$

Note that (22) is

$$
\tilde{x}=\beta x, \quad \tilde{y}=\alpha x+y,
$$




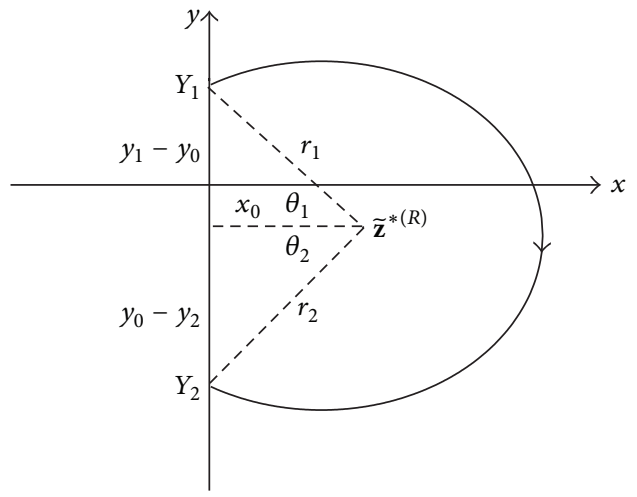

FIGURE 5: The right zone in the polar coordinate system.

so (22) is $y$-axis invariant, and the right half-system of (7) becomes

$$
\begin{aligned}
{\left[\begin{array}{c}
\dot{\tilde{x}} \\
\dot{\tilde{y}}
\end{array}\right] } & =T^{-1}\left[\begin{array}{l}
0 \\
\mu
\end{array}\right]+T^{-1}\left[\begin{array}{cc}
\tau_{R} & 1 \\
-\delta_{R} & 0
\end{array}\right] T T^{-1}\left[\begin{array}{l}
x \\
y
\end{array}\right] \\
& =\left[\begin{array}{l}
0 \\
\mu
\end{array}\right]+\left[\begin{array}{cc}
\alpha & \beta \\
-\beta & \alpha
\end{array}\right]\left[\begin{array}{c}
\tilde{x} \\
\tilde{y}
\end{array}\right] .
\end{aligned}
$$

The equilibrium is

$$
\widetilde{z}^{*(R)}=\left[\begin{array}{l}
x_{0} \\
y_{0}
\end{array}\right]=\left[\begin{array}{c}
\frac{\beta \mu}{\alpha^{2}+\beta^{2}} \\
-\frac{\alpha \mu}{\alpha^{2}+\beta^{2}}
\end{array}\right] .
$$

By using the polar coordinate transformation

$$
\begin{gathered}
r=\sqrt{\left(\tilde{x}-x_{0}\right)^{2}+\left(\tilde{y}-y_{0}\right)^{2}}, \\
\theta=\arctan \frac{\tilde{y}-y_{0}}{\tilde{x}-x_{0}} .
\end{gathered}
$$

Equation (25) then becomes

$$
\begin{aligned}
& \dot{r}=\alpha r, \\
& \dot{\theta}=-\beta .
\end{aligned}
$$

Rewrite (28) as

$$
\frac{d r}{d \theta}=-\frac{\alpha}{\beta} r
$$

which has the solution

$$
-\frac{\alpha}{\beta} \theta(t)=\ln r(t)+\gamma
$$

where $\gamma$ is a constant.

We will define a half-return map $\mathscr{P}$ from $\Sigma^{+}$to $\Sigma^{-}$by

$$
\mathscr{P}\left(y_{1}\right)=y_{2}
$$

such that for $Y_{1}=\left(0, y_{1}\right) \in \Sigma^{+}$there exists an orbit from $Y_{1}=\left(0, y_{1}\right)$ to $Y_{2}=\left(0, y_{2}\right) \in \Sigma^{-}$within the right zone.
In fact, we let the points $Y_{1}$ and $Y_{2}$ in the polar coordinate (27) be given by $\left(r\left(t_{1}\right) \theta\left(t_{1}\right)\right)$ and $\left(r\left(t_{2}\right), \theta\left(t_{2}\right)\right)$, respectively. From (30), it follows that

$$
\ln \frac{r\left(t_{2}\right)}{r\left(t_{1}\right)}=\frac{\alpha}{\beta}\left[\theta\left(t_{1}\right)-\theta\left(t_{2}\right)\right] .
$$

As shown in Figure 5, one has

$$
\begin{gathered}
r_{1}=r\left(t_{1}\right), \quad r_{2}=r\left(t_{2}\right) ; \quad \pi-\theta_{1}=\theta\left(t_{1}\right), \\
-\pi+\theta_{2}=\theta\left(t_{2}\right),
\end{gathered}
$$

where

$$
\begin{array}{cc}
r_{1}=\sqrt{x_{0}^{2}+\left(y_{1}-y_{0}\right)^{2}}, & r_{2}=\sqrt{x_{0}^{2}+\left(y_{0}-y_{2}\right)^{2}}, \\
\tan \theta_{1}=\frac{y_{1}-y_{0}}{x_{0}}, & \tan \theta_{2}=\frac{y_{0}-y_{2}}{x_{0}} .
\end{array}
$$

Then (32) becomes

$$
\ln \frac{r_{2}}{r_{1}}=-\frac{\alpha}{\beta}\left(\theta_{1}+\theta_{2}-2 \pi\right) .
$$

In general, for any $y_{1}>y_{0}>y_{2}$, let $\left(0, y_{1}\right)$ and $\left(0, y_{2}\right)$ be still given by $\left(r_{1}, \theta_{1}\right)$ and $\left(r_{2}, \theta_{2}\right)$, respectively, as shown in Figure 5. We mention that $\left(r_{1}, \theta_{1}\right)$ and $\left(r_{2}, \theta_{2}\right)$ are determined uniquely by $y_{1}$ and $y_{2}$ due to $0<\theta_{1}, \theta_{2}<\pi / 2$. Write

$$
\Lambda=\Lambda\left(y_{1}, y_{2} ; \xi\right)=\ln \frac{r_{2}}{r_{1}}+\frac{\alpha}{\beta}\left(\theta_{1}+\theta_{2}-2 \pi\right),
$$

where the parameter $\xi=(\alpha, \beta)$. If $\alpha$ and $\beta$ are fixed, we often omit the parameter $\xi$. From the above discussion, we see that

$$
\Lambda\left(y_{1}, y_{2}\right)=0
$$

if and only if (31) holds. That means that

$$
\Lambda\left(y_{1}, \mathscr{P}\left(y_{1}\right)\right)=0 .
$$

We point out that, by using (34), formula (36) becomes

$$
\begin{aligned}
\Lambda= & \frac{1}{2} \ln \frac{x_{0}^{2}+\left(y_{0}-y_{2}\right)^{2}}{x_{0}^{2}+\left(y_{1}-y_{0}\right)^{2}} \\
& +\frac{\alpha}{\beta}\left(\arctan \frac{y_{1}-y_{0}}{x_{0}}+\arctan \frac{y_{0}-y_{2}}{x_{0}}-2 \pi\right) .
\end{aligned}
$$

Next we consider the left zone governed by the saddle half-system $\mathbf{f}^{L}(\mathbf{z})$ in (7). If the nondegenerate homoclinic orbit $\Gamma$ exists, then, by using (20), it is not hard to check that the intersection points $C_{1}=\left(0, c_{1}\right)^{T}$ and $C_{2}=\left(0, c_{2}\right)^{T}$ are given by

$$
\begin{aligned}
c_{1,2} & =\frac{\mu}{\delta_{L}}\left(-\frac{\tau_{L}}{2} \mp \sqrt{\frac{1}{4} \tau_{L}^{2}-\delta_{L}}\right) \\
& =-\frac{\mu \lambda_{1,2}}{\delta_{L}}=-\frac{\mu \lambda_{1,2}}{\lambda_{1} \lambda_{2}}=-\frac{\mu}{\lambda_{2,1}} .
\end{aligned}
$$


So $C_{1} \in \Sigma^{+}$and $C_{2} \in \Sigma^{-}$. We mention that, from (24), one has $\widetilde{C}_{1,2}=C_{1,2}$.

From the discussion of (37) and (38), it follows that the existence of the homoclinic orbit $\Gamma$ is equivalent to $\mathscr{P}\left(c_{1}\right)=c_{2}$. Substituting for (39) by $y_{1}=c_{1}$ and $y_{2}=c_{2}$, we have

$$
\begin{aligned}
\Lambda= & \frac{1}{2} \ln \frac{x_{0}^{2}+\left(y_{0}-c_{2}\right)^{2}}{x_{0}^{2}+\left(c_{1}-y_{0}\right)^{2}} \\
& +\frac{\alpha}{\beta}\left(\arctan \frac{c_{1}-y_{0}}{x_{0}}+\arctan \frac{y_{0}-c_{2}}{x_{0}}-2 \pi\right) .
\end{aligned}
$$

By using (26), (34), and (40), a further simplification yields

$$
\begin{array}{r}
\Lambda\left(c_{1}, c_{2} ; \alpha, \beta\right) \\
=\frac{1}{2} \ln \frac{\lambda_{2}^{2}\left(\lambda_{1}^{2}-2 \alpha \lambda_{1}+\alpha^{2}+\beta^{2}\right)}{\lambda_{1}^{2}\left(\lambda_{2}^{2}-2 \alpha \lambda_{2}+\alpha^{2}+\beta^{2}\right)} \\
-\frac{\alpha}{\beta}\left(2 \pi-\arctan \frac{\lambda_{2} \alpha-\left(\alpha^{2}+\beta^{2}\right)}{\lambda_{2} \beta}\right. \\
\left.-\arctan \frac{\alpha^{2}+\beta^{2}-\lambda_{1} \alpha}{\lambda_{1} \beta}\right),
\end{array}
$$

which is just formula (16). The result is then obtained.

For the case $\mu<0$, the conclusion is similar; just exchange $L$ and $R$.

Remark 14. Note that (42) is independent of $\mu(\neq 0)$, so we may directly set $\mu=1$. In fact, from (12), (26), and (40), we find that $\mu(>0)$ only affects the size of trajectories.

Remark 15. From $0<\theta_{1}+\theta_{2}<\pi$, in (36), we find in the second term of (42) that

$$
\begin{array}{r}
\pi<2 \pi-\arctan \frac{\lambda_{2} \alpha-\left(\alpha^{2}+\beta^{2}\right)}{\lambda_{2} \beta} \\
-\arctan \frac{\alpha^{2}+\beta^{2}-\lambda_{1} \alpha}{\lambda_{1} \beta}<2 \pi .
\end{array}
$$

On the other hand, in the first term of (42), one has

$$
\begin{aligned}
\Delta & \equiv \lambda_{2}^{2}\left(\lambda_{1}^{2}-2 \alpha \lambda_{1}+\alpha^{2}+\beta^{2}\right)-\lambda_{1}^{2}\left(\lambda_{2}^{2}-2 \alpha \lambda_{2}+\alpha^{2}+\beta^{2}\right) \\
& =\left(\lambda_{1}-\lambda_{2}\right)\left[2 \alpha \lambda_{1} \lambda_{2}-\tau_{L}\left(\alpha^{2}+\beta^{2}\right)\right]
\end{aligned}
$$

where $\tau_{L}=\lambda_{1}+\lambda_{2}$. From (43) and (44), we find that the existence of a nondegenerate homoclinic loop implies $\tau_{L} \tau_{R}<$ 0, as stated in Proposition 10.

Remark 16. In particular, we consider the saddle-center system, where $\alpha=0$. From (42), one has

$$
\Lambda=\frac{1}{2} \ln \frac{\lambda_{2}^{2}\left(\lambda_{1}^{2}+\beta^{2}\right)}{\lambda_{1}^{2}\left(\lambda_{2}^{2}+\beta^{2}\right)} .
$$

From

$$
\Delta=\lambda_{2}^{2}\left(\lambda_{1}^{2}+\beta^{2}\right)-\lambda_{1}^{2}\left(\lambda_{2}^{2}+\beta^{2}\right)=\tau_{L}\left(\lambda_{2}-\lambda_{1}\right) \beta^{2},
$$

it is easy to see that $\Lambda<0,=0$ and $>0$ are equivalent to $\tau_{L}<0,=0$ and $>0$, respectively.

On the other hand, it is not hard to check that if $\Lambda=0$ and $\tau_{L}=0$, then $\alpha=0$. In this case, all the trajectories inside the homoclinic loop are closed around the center.

Remark 17. It is easy to see that $y_{2}=\mathscr{P}\left(y_{1}\right)$ is a decreasing function of $y_{1}$. From (38), $\Lambda\left(y_{1}, \mathscr{P}\left(y_{1}\right)\right)=0$, we have

$$
\frac{\partial \Lambda}{\partial y_{1}}+\frac{\partial \Lambda}{\partial y_{2}} \mathscr{P}^{\prime}\left(y_{1}\right)=0 .
$$

So $\partial \Lambda / \partial y_{1}$ and $\partial \Lambda / \partial y_{2}$ have the same sign.

Let $y_{3}<y_{2}$ and $\left(0, y_{3}\right)$ be given by $\left(r_{3}, \theta_{3}\right)$; then $r_{3}>r_{2}$, $\theta_{3}>\theta_{2}$. Let $\alpha \geq 0$. From (38) and (36), it follows that $\Lambda\left(y_{1}, y_{3}\right)>\Lambda\left(y_{1}, y_{2}\right)$. Moreover, by (38), one has

(1) $\Lambda\left(c_{1}, c_{2}\right)<0$ if and only if $\mathscr{P}\left(c_{1}\right)<c_{2}$,

(2) $\Lambda\left(c_{1}, c_{2}\right)=0$ if and only if $\mathscr{P}\left(c_{1}\right)=c_{2}$,

(3) $\Lambda\left(c_{1}, c_{2}\right)>0$ if and only if $\mathscr{P}\left(c_{1}\right)>c_{2}$.

3.2. Degenerate Case. For $\mu=0$, the origin is the only equilibrium of both half-systems. If the system has twofold node with opposite stability, then, as pointed in Freire et al. [6], there exists a continuum of homoclinic loops, consisting of degenerate homoclinic orbits. Conversely, the existence of degenerate homoclinic orbits implies that the equilibrium is neither saddle nor focus (or nor center). So one has the following result.

Theorem 18. If $\mu=0$, then the existence of homoclinic orbits for (7) is equivalent to

$$
\tau_{L} \tau_{R}<0, \quad 0<\delta_{i}<\frac{1}{4} \tau_{i}^{2}, \quad i=L, R .
$$

Under the conditions (48), the continuum of homoclinic loops is above the $x$-axis for $\tau_{L}>0$ and $\tau_{R}<0$ and under the $x$-axis for $\tau_{L}<0$ and $\tau_{R}>0$.

Remark 19. For a continuum of homoclinic loops, see Example 2. In fact, by calculating the eigenvalue (19) with the corresponding eigenvectors (20), one may find that all the homoclinic loops lie between an unstable manifold and a stable manifold.

\section{Homoclinic Bifurcations}

Based on the study of homoclinic orbits in the last section, now the homoclinic bifurcations in (7) are discussed. We first provide the generic homoclinic bifurcation related to nondegenerate homoclinic orbits and then study the problem of discontinuity induced homoclinic bifurcations. The latter will be divided into two cases to study: nondegenerate and the degenerate. 


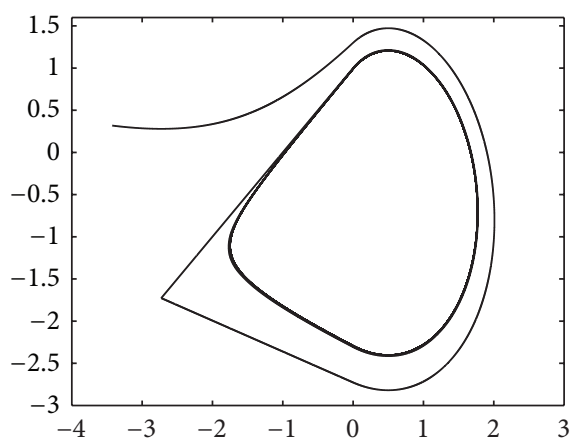

(a)

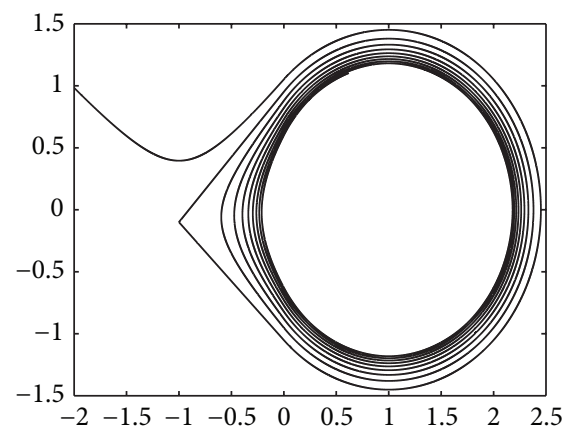

(a)

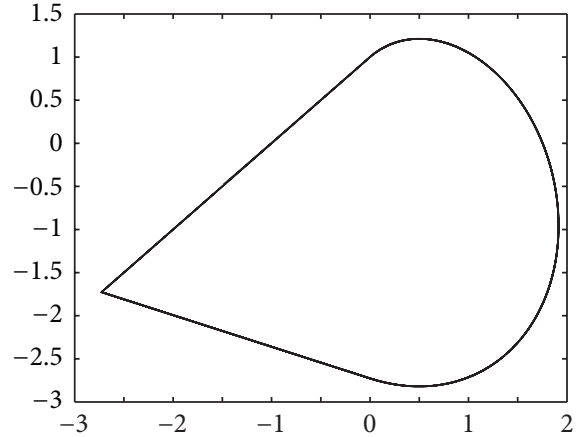

(b)

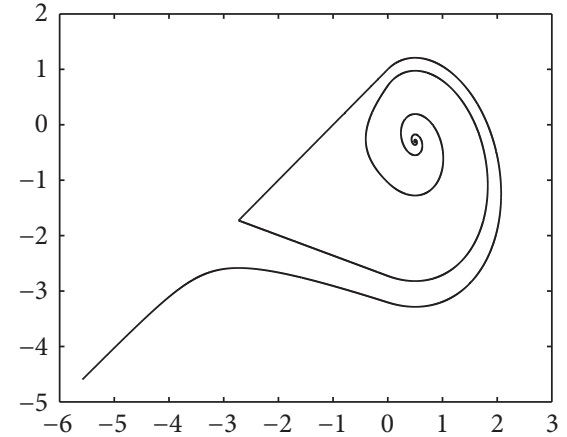

(c)

FIgURE 6: Bifurcation of saddle-focus systems.

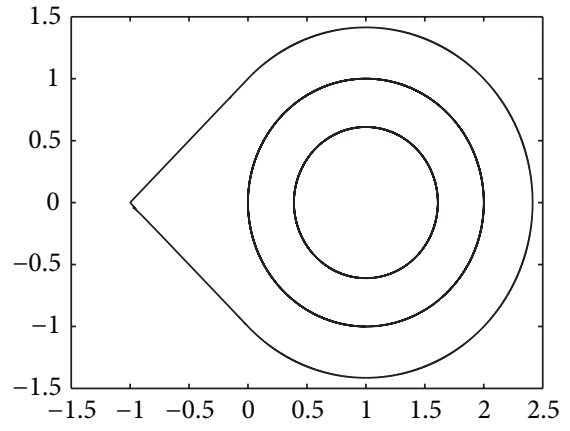

(b)

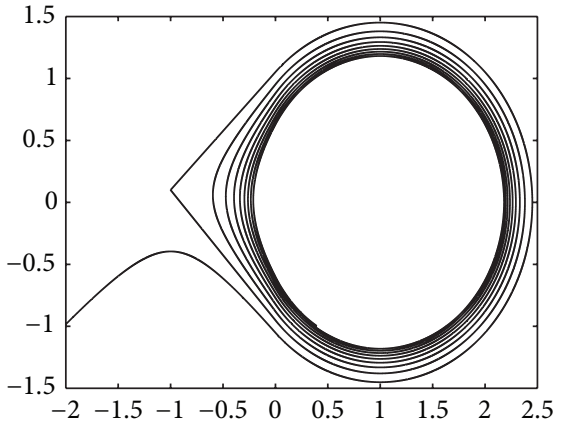

(c)

FIgURE 7: : Bifurcation of saddle-center systems.

4.1. Generic Homoclinic Bifurcations. In this subsection, we discuss bifurcations related to the nondegenerate homoclinic orbits, called generic homoclinic bifurcations. For simplicity, we still assume that the left half-system in (7) is saddle, so $\mu>0$. Two kinds of homoclinic bifurcations are studied: for the saddle-focus system and for the saddle-center system.

For the saddle-focus system, $\tau_{R}=2 \alpha \neq 0$. As we saw in Theorem 13 and Remark 15, the existence of a nondegenerate homoclinic loop depends on $\Lambda=0$ and $\tau_{L} \tau_{R}<0$. Varying the parameters related to $\Lambda$ slightly, the homoclinic loop may disappear.

(1) When $\tau_{R}>0$, the right zone is governed by an RF system. From Remark 17 and the analysis of the phase portraits, we find that, for $\Lambda\left(c_{1}, c_{2}\right)<0$, one has $\mathscr{P}\left(c_{1}\right)<c_{2}$ and there is no periodic orbits in the system and that, for $\Lambda\left(c_{1}, c_{2}\right)>0$, one has $\mathscr{P}\left(c_{1}\right)>c_{2}$ and there is an attracting limit cycle around the focus.

(2) When $\tau_{R}<0$, in the right zone is an AF system. For $\mathscr{P}\left(c_{1}\right)>c_{2}$, there is no periodic orbits in the system. For $\mathscr{P}\left(c_{1}\right)<c_{2}$, there is a repelling limit cycle around the focus.

We mention here that the system has at most one limit cycle by Proposition 9 .
Example 20. A bifurcation of saddle-focus systems is shown as in Figure 6, where the parameters in (8) are $\mu=1, \tau_{L}=$ $-0.6333, \delta_{L}=-0.3667$, and $\delta_{R}=2$, and we take $\tau_{R}=0.4$ for (a), 0.5 for (b), and 0.6 for (c). There is a limit cycle in Figure 6(a).

Now we discuss the saddle-center system case; that is, $\tau_{R}=0$.

(1) For $\tau_{R}=0$ vary $\tau_{L}$ and $\delta_{L}$, there exists a limit cycle in the right zone tangent to the $y$-axis. It is the unique limit cycle.

(2) For $\tau_{L}$ and $\delta_{L}$ fixed vary $\tau_{R}$, in the right zone is a focus system, no limit cycle in this case.

(3) Varying $\tau_{L}, \delta_{L}$, and $\tau_{R}$, it turns into the saddle-focus system case.

Example 21. A bifurcation of saddle-center systems is shown in Figure 7, where the parameters are $\mu=1, \delta_{L}=-1, \tau_{R}=0$, and $\delta_{R}=1$, and take $\tau_{L}<0$ for (a), $\tau_{L}=0$ for (b), and $\tau_{L}>0$ for (c).

In order to summarize the above conclusions we let $\mu>0$, $\beta>0$, and $\delta_{L}<0$ be fixed and consider the $\left(\tau_{R}, \tau_{L}\right)$-plane, here $\tau_{R}=2 \alpha$ and $\tau_{L}=\lambda_{1}+\lambda_{2}$. Figure 8 is the homoclinic bifurcation diagram for the nondegenerate case. The line $\Lambda=$ 0 divides the plane into two parts: upper is for $\Lambda>0$ and 


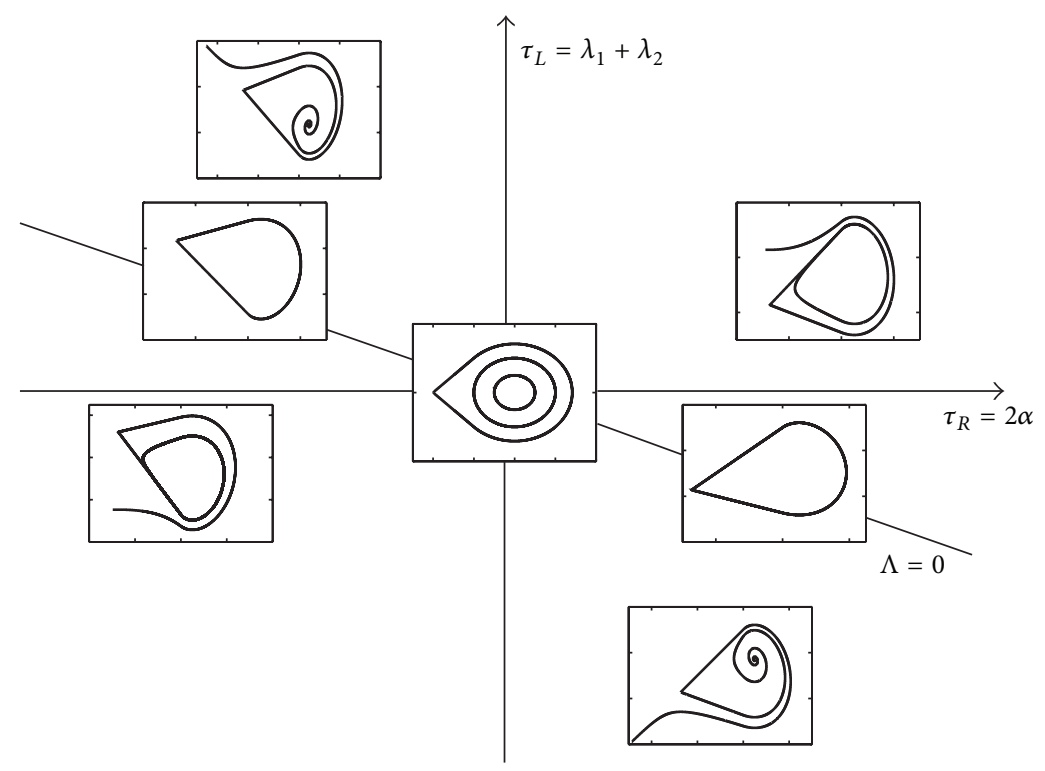

FIGURE 8: A bifurcation diagram.

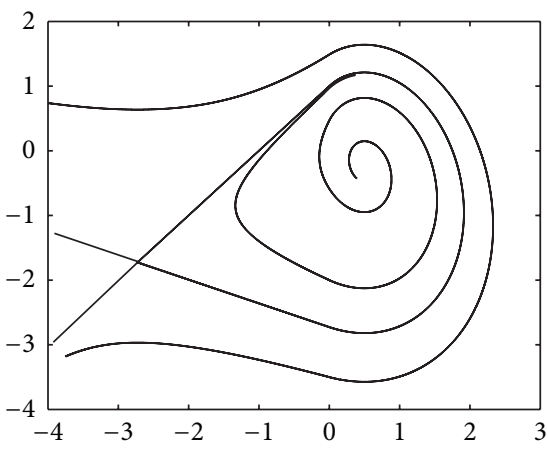

(a)

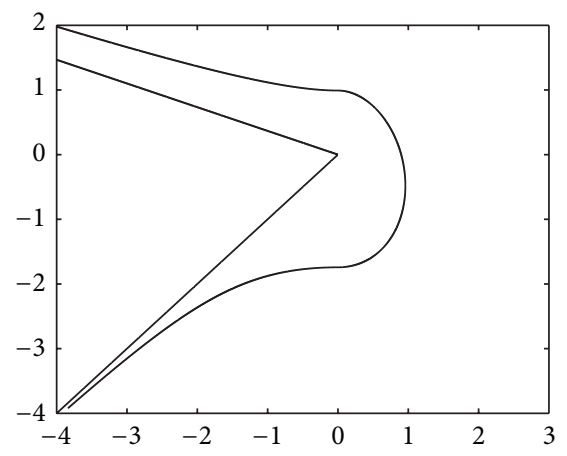

(b)

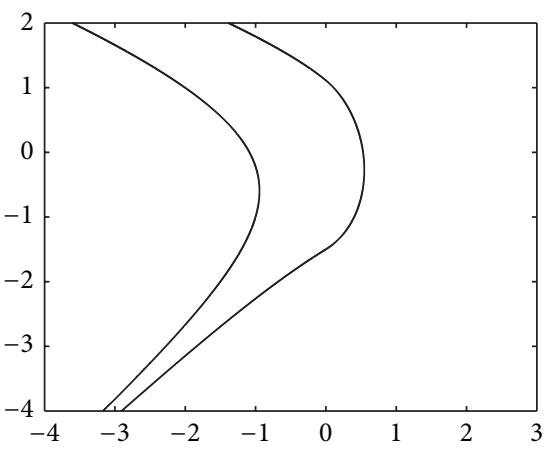

(c)

FIGURE 9: Discontinuity induced homoclinic bifurcation in the nondegenerate case.

bellow is for $\Lambda<0$. All the trajectories are clockwise. From Figure 8 , we can find that the parameters $\tau_{R}$ and $\tau_{L}$ unfold all possible generic homoclinic bifurcation behaviors.

4.2. Discontinuity Induced Homoclinic Bifurcations. In this subsection, we study the problem of the discontinuity induced homoclinic bifurcations (DIHBs) related to variation of boundary equilibria. Herein we take $\mu$ as a bifurcation parameter and discuss two kinds of DIHBs: the nondegenerate case and the degenerate case.

4.2.1. Nondegenerate Case. We still assume that the equilibrium of (7) in the left zone is a visible saddle for $\mu>0$, and then the right zone has a visible focus (or center). It is obvious that whenever $\mu=0$ both of the visible equilibria will coincide on the nonsmooth boundary $y$-axis and become invisible as $\mu<0$, which is a nonsmooth fold boundary equilibrium bifurcation; see also [1]. From the discussion in Section 3, the homoclinic orbits exist for $\Lambda=0$ and shrink to an equilibrium gradually as the scale parameter $\mu$ decreases from positive to 0 then disappear as $\mu<0$. This is the discontinuity induced homoclinic bifurcation in the nondegenerate case.

Example 22. Following Example 1(a), set $\tau_{L}=-0.6333, \delta_{L}=$ $-0.3667, \tau_{R}=0.5$, and $\delta_{R}=2$ in (7) and (8). Take $\mu$ near zero. Figures 9(a), 9(b), and 9(c) show a discontinuity induced homoclinic bifurcation of the system for $\mu>0, \mu=0$, and $\mu<0$, respectively.

In Figure 9, we find that, for $\mu>0$, the system is saddlefocus with a nondegenerate homoclinic orbit as shown in (a), that, for $\mu=0$, two equilibria coincide at the origin and the homoclinic orbit becomes a broken line as in (b), and that for $\mu<0$ both equilibria become invisible and the homoclinic orbits disappear as in (c).

4.2.2. Degenerate Case. As mentioned in Section 3, under the conditions of Theorem 18 there exists a continuum of 


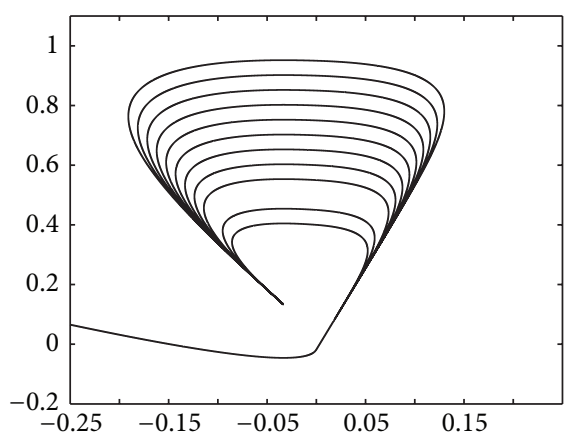

(a)

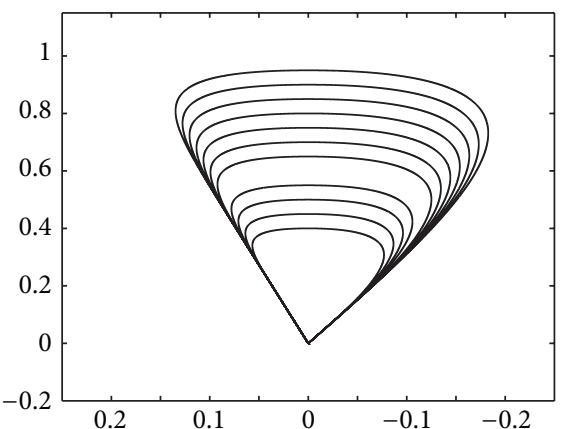

(b)

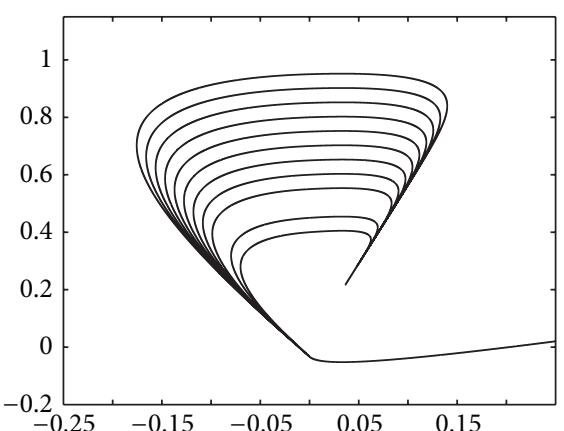

(c)

Figure 10: Discontinuity induced homoclinic bifurcation in the degenerate case.

homoclinic loops when $\mu=0$. Varying the parameter $\mu$, the system (7) has a type of boundary equilibrium bifurcation, called persistent-node bifurcation. Here as the bifurcation parameter $\mu$ crosses the critical value 0 , the visible node of one half-system in (7) becomes invisible, and the other invisible node becomes visible, while all homoclinic orbits disappear for $\mu \neq 0$. This is a discontinuity induced homoclinic bifurcation in the degenerate case.

Example 23. Following Example 2, for (7) and (8), set $\tau_{L}=4$, $\delta_{L}=3, \tau_{R}=-6$, and $\delta_{R}=3$. We take $\mu$ near zero. Figures 10(a), 10(b), and 10(c) show the discontinuity induced homoclinic bifurcation for degenerate case for $\mu=-0.1$, $\mu=0$, and $\mu=0.1$, respectively.

\section{Conclusions}

We have studied in detail the homoclinic bifurcations in the planar piecewise-linear systems with two zones. Firstly, the system is transformed to a canonical form. Then both nondegenerate and degenerate homoclinic orbits are investigated. The necessary and sufficient conditions for the existence of both a nondegenerate homoclinic orbit and a continuum of degenerate homoclinic orbits are established for discussion of the homoclinic bifurcation problem. A generic homoclinic bifurcation diagram is shown as in Figure 8. We have found that there are two kinds of DIHBs, one is nondegenerate and the other is degenerate. Nonsmooth fold boundary equilibrium bifurcation occurs accompanying DIHB in nondegenerate case, and persistent-node boundary equilibrium bifurcation occurs accompanying DIHB in degenerate case. To sum up, at least two parameters are needed to unfold all possible homoclinic bifurcations.

\section{Acknowledgment}

This work was supported by the NNSF of China (Grant nos. 11072274 and 11272169).

\section{References}

[1] M. di Bernardo, C. J. Budd, A. R. Champneys, and P. Kowalczyk, Piecewise-Smooth Dynamical Systems, vol. 163 of Applied Mathematical Sciences, Springer, London, UK, 2008.
[2] M. di Bernardo, D. J. Pagano, and E. Ponce, "Nonhyperbolic boundary equilibrium bifurcations in planar Filippov systems: a case study approach," International Journal of Bifurcation and Chaos in Applied Sciences and Engineering, vol. 18, no. 5, pp. 1377-1392, 2008.

[3] R. Lum and L. O. Chua, "Global properties of continuous piecewise linear vector fields-part I: simplest case in $\mathbb{R}^{2}$," International Journal of Circuit Theory and Applications, vol. 19, no. 3, pp. 251-307, 1991.

[4] E. Freire, E. Ponce, F. Rodrigo, and F. Torres, "A piecewise linear electronic circuit with a multiplicity of bifurcations," International Journal of Bifurcation and Chaos in Applied Sciences and Engineering, vol. 14, no. 11, pp. 3871-3881, 2004.

[5] D. Simpson, Bifurcations in Piece-Wise Continuous Systems, World Scientific, 2010.

[6] E. Freire, E. Ponce, F. Rodrigo, and F. Torres, "Bifurcation sets of continuous piecewise linear systems with two zones," International Journal of Bifurcation and Chaos in Applied Sciences and Engineering, vol. 8, no. 11, pp. 2073-2097, 1998.

[7] D. J. W. Simpson and J. D. Meiss, "Andronov-Hopf bifurcations in planar, piecewise-smooth, continuous flows," Physics Letters A, vol. 371, no. 3, pp. 213-220, 2007.

[8] C. D. Mitrovski and Lj. M. Kocarev, "Periodic trajectories in piecewise-linear maps," IEEE Transactions on Circuits and Systems. I., vol. 48, no. 10, pp. 1244-1252, 2001.

[9] J. M. Gonçalves, "Regions of stability for limit cycle oscillations in piecewise linear systems," IEEE Transactions on Automatic Control, vol. 50, no. 11, pp. 1877-1882, 2005.

[10] Y. Xiong and M. Han, "Bifurcation of limit cycles by perturbing a piecewise linear Hamiltonian system," Abstract and Applied Analysis, vol. 2013, Article ID 575390, 19 pages, 2013.

[11] F. Liang, M. Han, and V. G. Romanovski, "Bifurcation of limit cycles by perturbing a piecewise linear Hamiltonian system with a homoclinic loop," Nonlinear Analysis: Theory, Methods \& Applications, vol. 75, no. 11, pp. 4355-4374, 2012.

[12] R. I. Leine and H. Nijmeijer, Dynamics and Bifurcations of Non-Smooth Mechanical Systems, vol. 18 of Lecture Notes in Applied and Computational Mechanics, Springer, Berlin, Germany, 2004.

[13] L. Perko, Differential Equations and Dynamical Systems, vol. 7 of Texts in Applied Mathematics, Springer, New York, NY, USA, 3rd edition, 2001. 


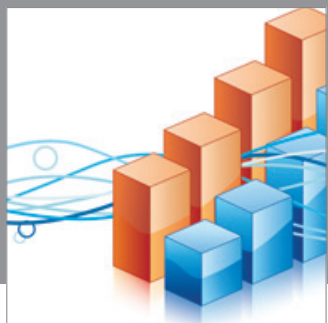

Advances in

Operations Research

mansans

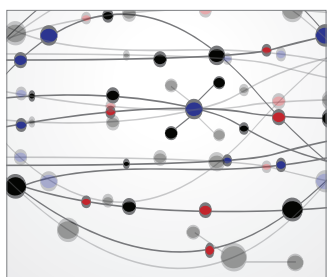

The Scientific World Journal
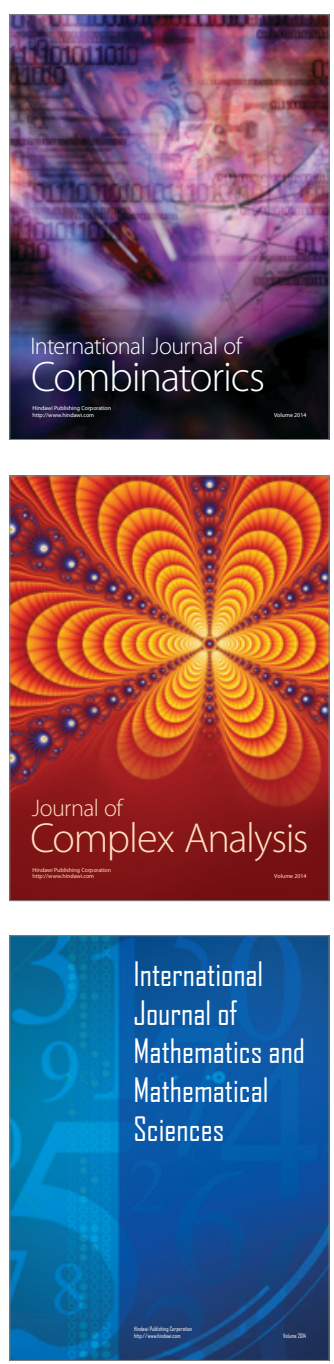
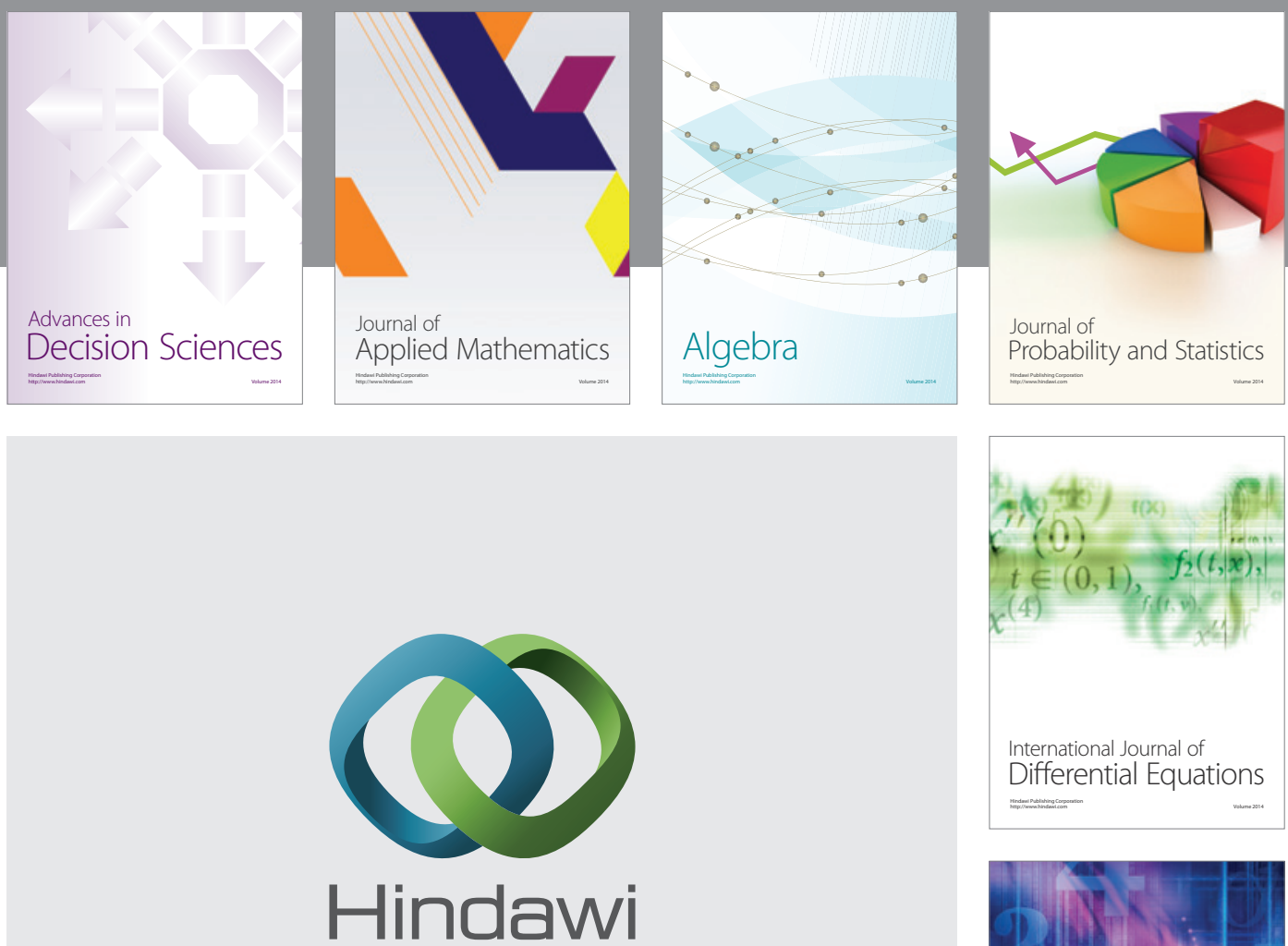

Submit your manuscripts at http://www.hindawi.com
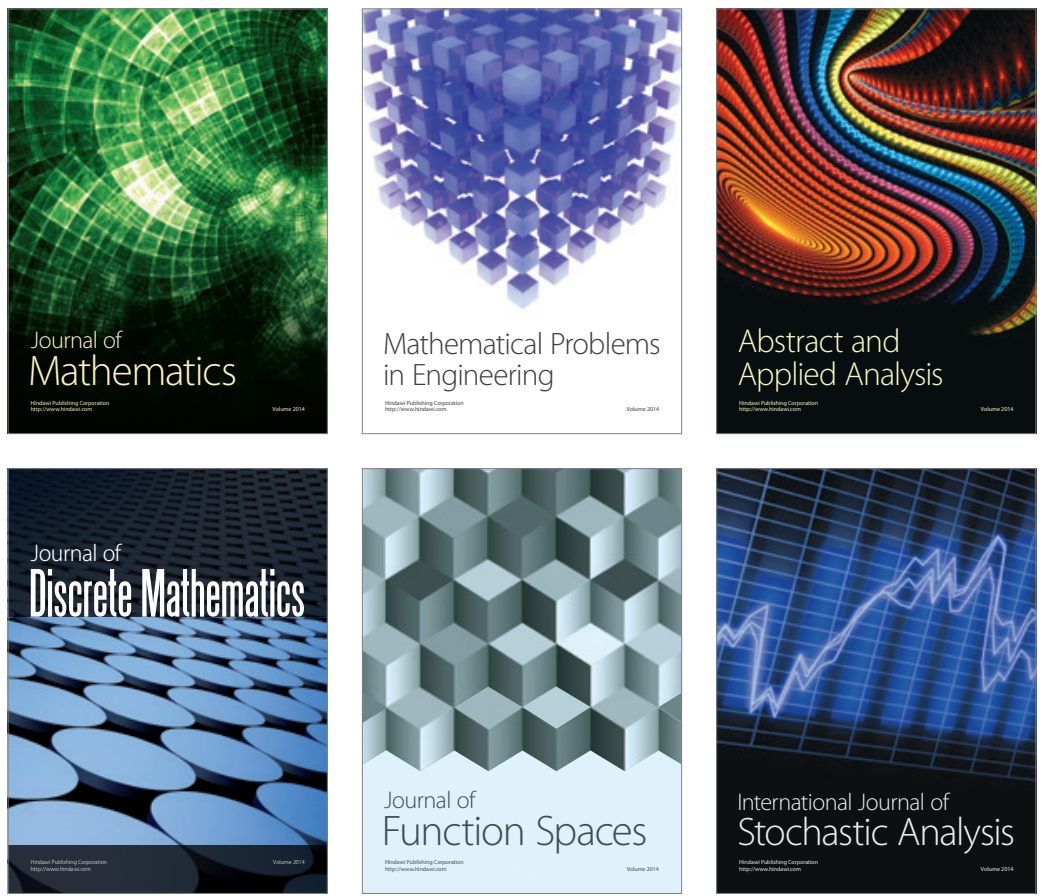

Journal of

Function Spaces

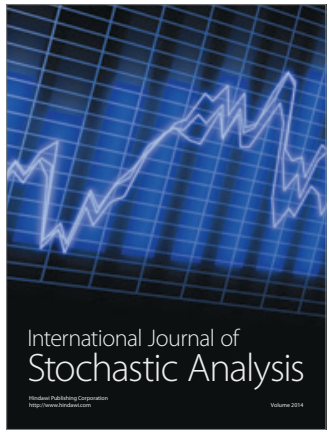

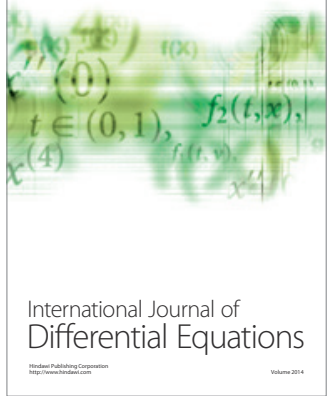
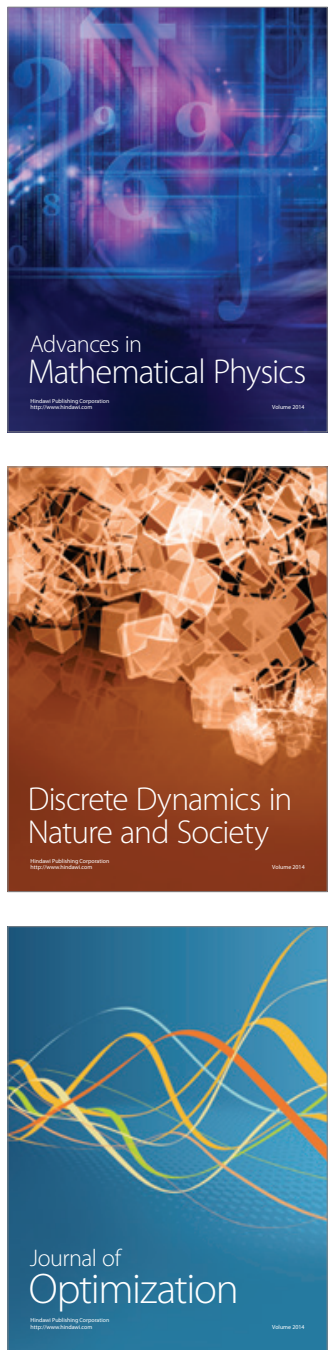